\title{
Recovery of global symmetries in a 't Hooftian universe
}

\author{
Alexandre Furtado Neto*
}

UNESP Alumnus

\begin{abstract}
A new charge is postulated in a finite, closed, Euclidean discrete space to restore all fundamental symmetries on a global level. Gravity emerges as a residual effect of the electromagnetic force in this scenario, resulting in a deterministic toy universe driven by a single input parameter. Randomness is identified using a Chaintin argument. Aleph0 definite value is tied to the size of the universe. This is not an interpretation of Quantum Mechanics, but a deeper attempt to describe nature.
\end{abstract}

PACS numbers: $12.10 . \mathrm{Kt}$

Keywords: cellular automaton, nonlocality, emerging gravity, unification, cardinality

\section{Introduction}

Wheeler [1] coined the aphorism 'it from bit'. With this, he meant that anything physical, any it, derives its existence from discrete binary choices, or bits. This gives support to the notion that information has an ontological nature. The concept implies that physics, particularly quantum physics, isn't really about reality, but just our best description of what we observe.

In this regard, cellular automata (CAs) are mathematical idealizations of physical systems in which space and time, an evolution parameter, are discrete. Their attractiveness comes from the notion that simple rules can lead to very complex behavior, tending to long and interesting evolutions.

An alternative representation of the universe is developed in this work using the cellular automaton paradigm. The theme has been explored for a long time (see $[2,3,4,5,6,7,8$, $9,10,11,12]$ for example). However, these studies generally remain in the abstract realm or present very limited models. Here, a full $\mathbf{4}+\mathbf{1}$ core specification is posited. Although it is a qualitative analysis for the time being, the model is amenable to immediate computational investigation.

Just classical logic and plain integer math, along with a hint of topology, are used in the dynamics.

It will be clear that foundationally this is not an interpretation of Quantum Mechanics, but a deeper attempt to describe nature.

*alexandre.com@yahoo.com 


\section{Space and time}

A collection of $2 L$ coexisting $S I D E^{3}$-sized Euclidean lattices each, closed on themselves as three-dimensional tori, represents an absolute inertial reference frame. Each cell contains an equal amount of information (a register) and are synchronized by a common time $t \in \mathbb{N}$, exchanging information with its nine neighbors only (six in dimensions $x y z$, two in dimension $u$ and one in dimension $v$ ). This exchange is done alternately, so time homogeneity is recovered at each two clock ticks, configuring a cellular automaton.

The single input parameter SIDE was estimated from the size of the observable universe at a fraction $(1 / 8)^{1}$ of the Planck scale as

$$
\begin{aligned}
\text { SIDE } & =2^{\text {order }}, \\
& \approx 6.582 \times 10^{63},
\end{aligned}
$$

where order $=212$. The arithmetic uses SIDE as module.

The torus is the simplest topological structure capable of holding a 3D universe and charge quantization. The finitude of the universe is a necessary condition to reach this quantization (Smith [13]). Cosmological observations favor a multi-connected rather than a simply connected universe (see Lachieze-Rey and Luminet [14]).

\section{Bubbles}

Each register contains six bits of charge $d, c_{2}, c_{1}, c_{0}, w, q$, a few integer variables, e.g., $b, f, \phi, \varphi$, noise, and four integer vectors $\boldsymbol{p}, \boldsymbol{s}, \boldsymbol{o}, \boldsymbol{p o l e}$. Most properties spread to the expanding cells, with the exception of the vector $\boldsymbol{p}$, which is passed to a single cell in its path.

A bubble is an expanding spherical wavefront of information organized on the registers. Whenever vector $\boldsymbol{p}$ moves, it casts such a perturbation. The von Neumann neighborhood Dirs is used in a Case, Rajan and Shende algorithm from [15]. There they show that the accommodation of the wavefront takes $2 D$ clock ticks (one light step), where $D$ is the cube diagonal, so bubbles expand with the same speed $c$, increasing $|\boldsymbol{o}|$ in the occupied registers. In some cases, concurrent access to a common cell is attempted, being per se an undecidable problem. A tree algorithm is then used to overcome this limitation and select a path. Incidentally, this direction index behaves like a modulo 6 random number, being the basis for decision making in interactions (see Chaitin [16]).

A bubble is eventually reissued from a point calculated on its surface by resetting the origin vector $(\boldsymbol{o}=\mathbf{0})$ when interacting with other bubbles. One extra variable named code is used to qualify bubble superpositions.

Each bubble lives in a separate $3 \mathrm{~d}$-space $L_{i}, i=\{1 \ldots 2 L\}$ to avoid propagation issues. $F$ superposing bubbles with a common bond $b_{j}=B, j=\{1 \ldots F\}$, have frequency $f_{j}=F$. Two such bubbles with some or all opposite charges is called a pair.

Bubbles also have a sinusoidal phase $\phi$ coupled to $f$, achieved by a recurrent algorithm. Together with the related variable $\varphi$, they implement interference and self-interference (see Sciarretta [11]). Each occupied register has $\varphi=\phi$, which decays as

$$
\varphi^{k+1}=\varphi^{k}\left(1-\frac{1}{2 k}\right)
$$

\footnotetext{
${ }^{1}$ The exact granularity will be obtained when it is verified that the model generates the same number of particles present in the universe.
} 
at each light step $k$. During wavefront accommodation the value of noise is updated using the selected direction index (Dirs[index $]$ ) and latter compared with $|\phi+\varphi|$, which indicates whether a bubble-bubble interaction is legal - this produces the observed harmonic behavior at the ensemble level. Momentun $\boldsymbol{p}$ is moved to the cell that minimizes the pole vector.

Charges are represented by bits. The new dualitat charge $d$ was introduced to preserve symmetries on a global level. The electric charge $q$ is associated as ever with attraction/repulsion. The three color charges $c_{2}, c_{1}, c_{0}$ enforce the strong force structure. Let signature be $\operatorname{sig}=c_{2}+c_{1}+c_{0}$. A bubble is matter $M$ if sig $<2$, or antimatter $\bar{M}$, otherwise. Also, it is neutral $N$, if $s i g=0$ or anti-neutral $\bar{N}$, if $\operatorname{sig}=3$. The definition color $=2^{2} c_{2}+2^{1} c_{1}+2^{0} c 0$ will be used latter. On the other hand, the weak charge $w$, or chirality, is associated with congruence.

Vector $\boldsymbol{p}$ is related to motion direction, while vector $\boldsymbol{s}$ to spatial rotation. I will refer to them as momentum and spin for convenience. The cell on the surface of the bubble pointed by vector $\boldsymbol{p}$ from its own center is defined as its pole.

Finally, a loose association between the terms bubble and (unit of) energy is assumed.

\section{Interactions}

\subsection{Basic rules}

Interactions are evaluated at the last tick of the wavefront accommodation. Prior to interaction, the frequency and noise variables of the bubbles are updated. Superposing bubbles are classified according to their charge content.

When a bubble is reissued after interacting with another bubble and, in addition, their poles coincide, all bonded bubbles are also reissued, characterizing an ontological collapse. The possibility of the collapsing parts having a space-like separation is supported by the fact that the phase of accommodation of the wavefront lasts $2 D$ ticks, sufficient for the information to propagate to all regions of the lattice.

\subsection{Interactions}

The detailed algorithmic description of all interaction rules is contained in the Appendix. Half of the cells are active, while the other half are passive, alternating roles in time. The basic loop consists of the following steps

\section{- Commute}

The active/passive roles are exchanged and data from the active cells are copied to the passive cells.

\section{- Update}

Passive cells are shifted in the $v$ dimension and compared until a complete loop of $S I D E^{2}$ steps. Properties such as the frequency are updated.

\section{- Interact}

After having data being copied back to the active cells, again, the passive cells are shifted in the $v$ dimension and compared for possible interactions, which are encoded in the code variable.

\section{- Expand}

All bubbles expand one light step in their respective $x y z$ sub-lattice. When momentum $\boldsymbol{p}$ moves, it releases a new perturbation wavefront from its old position. 


\subsection{Initial state}

Let $S$ be the number of states resultant of the combination of all register values, all positions in the lattice and all bubbles $L$. From any state $k$, the system eventually enters an endless period loop $T_{k}$ with definite net Shannon entropy. The real world corresponds to one of these loops with $T_{R} \ll S$.

Among the countless possibilities, I choose the following platonic solution for the initial state problem. A total of $L \equiv S I D E^{2}$ bubbles are uniformly distributed on the $z=0$ plane of the lattice at start up, the hologram. Since $S I D E \bmod 3=1$, one single row of the hologram has all $s_{x}=s_{y}=0, s_{z}= \pm S I D E / 2$ and $d=s_{z} \geq 0$, inducing one topologically trapped magnetic monopole, per sector, in line with Smith [13]. The momentum vectors, in turn, receive the value $\boldsymbol{p}=\boldsymbol{k} \times \boldsymbol{s}$, where $\boldsymbol{k}$ is the $z$ direction.

We are done with the axiomatic part by now. In the remainder of the paper, the arguments give support to the choices made and compose a minimalist interpretation, carefully avoiding unnecessary speculation.

\subsection{Evolution}

The system evolves through this unphysical era until it reaches a stationary sequence of states, the very very long physical cycle of period $T_{R}$, or Poincaré cycle. During the unphysical era, most bubbles are annihilated, creating a vacuum, both in Orbis, the sector with $d=0$, or in the Dark Sector, with $d=1$. The remaining bubbles within each sector, segregate on islands of same size with positive or negative charges (charge quanta) due to the aforementioned monopole. Antimatter tends to migrate to the Dark Sector due to inter sector interactions. Observe that masses from Orbis are not seen from the Dark Sector and vice versa.

\subsection{Packets}

Each region of space contains a homogeneous distribution of electrically neutral superposing bubbles (zero-point energy, or a vacuum), basically photons and $\mathrm{Z}$ particles, but also the charged W bosons, whether completely or partially formed. Some of these bubbles are recruited via the messenger interaction above, being dynamically added to a local packet of bonded bubbles. If the packet contains colored or neutral bubbles with $f=1$, different pairs will be captured from the vacuum.

Meanwhile, other bubbles wander away from the packet (dissipation) such that, when combined with the inherent non-linearity of the underlying system, an equilibrium situation is reached including many propellers (see definition below), configuring in this way the mass of the packet. The non-equilibrium caused by nearby charges or masses can change the motion of the packet.

Packets possessing a quantized set of bubbles with $f=1$ will have a collective "fermionic" character, while the pairs overlaid with anti-aligned spins give a packet a "bosonic" character, distinguished by their charge balance. The exception are the neutrino fragments, formed as $N N(\nu)$ or $\bar{N} \bar{N}(\bar{\nu})$ with their aligned spins, which, even possessing $f=2$, also present a "fermionic" character. In other words, if the packet includes a (quantized) population of bubbles with $f=1$ identical charge $q$ then we have a fermion. Rather, if the population is formed by equal net weak charge, we have a neutral massive boson, and if the pairs also have non trivial electric balance then we have a charged weak boson. If, diversely, color is envolved and $f=1$, we have a quark fragment. Finally, if these bubbles are combined in pairs with the same non-neutral color, then we have a gluon fragment. The exception to the rule is the photon, which is an expading shell 'packet'. 
Ephemeral resonance states, including radial vibration modes $^{2}$ (see Itô [17]) induced by the presence of neutrinos (which also help to conserve angular momentum), are also possible, adding to the formation of a mass spectrum.

The Hofer effect is the expected tendency for all spins of a packet to align radially either inward or outward (spin up/down), as predicted in Hofer [18]. In that work, there is an explanation of how magnetic effects emerge from symmetry breaking of this spherical pattern.

\subsection{Long range energy exchange}

A pure photon $\gamma$ is a multipair $(f=2 n, n>0)$ where each pair has all its charges in opposition. A photon fragment bonded to a packet is dubbed a propeller.

The long range behavior of photons is due to the absence of other bubbles with the same $b$ value to allow an inertial interaction (not a propeller), interacting instead either as a light-matter scattering or a refraction process above.

It may happen that two bubbles in a packet, when interacting as above, are re-emmited from the same point, that is, their poles coincide, so a pair is formed. These coincidences may repeat so that overlapping of many bubbles having a common $b$ value becomes possible. If they move away stimulated by another bubble-bubble process, a photon is released - spontaneous emission being a special case for faint fields. In other words, photons are normally released in an atomic electronic decay, shaped by the spherical harmonics originating from charge quantization. This is the main explanation for the ubiquitous presence of all sort of quanta. The primary quantized quantity, is truly the electric charge.

Photons also come similarly from bremsstrahlung. In this scenario, the spectrum is continuous since it has nothing to do with spherical harmonics.

The messenger versus bubble interaction also implies that photons may lose components while traveling (photon aging) thereby supporting General Relativity observations and the CMB.

\subsection{Weak decay}

No randomness is involved in the calculation of the interactions, so the model is in fact deterministic, but the ubiquituous presence of weak charges imply in an apparently random decay of particles. The $W^{-}$and $Z$ bosons, even if not completely formed (virtual particles?), are responsible for it.

\subsection{Higher order particle structures}

When reorganizing after a collapse, bosons and fermions can be formed in a variety of ways, spins can be flipped etc. With all these ingredients, bound states of the strong and electromagnetic forces are a natural consequence.

\section{Discussion}

G. 'tHooft [12] shows that systems of this type can be associated to a permutation operator and, therefore, can be mapped to a large Hilbert space. Thus, at least in principle, all the machinery of operator mechanics, in particular the Schrodinger equation, can be used to analyze them - the point here is that the system certainly presents a quantum behavior.

\footnotetext{
${ }^{2}$ Empirically in three generations of leptons and quarks.
} 
In this context, Born's rule arises naturally without the need to be postulated, bringing with it quantum probabilities. Perhaps new analysis tools should be devised to explore the model in search of physics beyond the Standard Model.

Last but not least, a gravity-like dynamics emerges as a residual effect of the electromagnetic force (see also Assis [19]) - the halos formed in the sea of messengers around the masses caused by the absorption of messengers (perceived as not shielding because of the relatively low number of messengers absorbed) causes an imbalance in the distribution of momentum, resulting in an always attractive phenomenon, or gravity.

The model is non-local under the light-time basis, though being strictly local, but no signaling is possible at any classical limit. An intuitive argument allows us to associate $S I D E$ to the much sought for cardinality of natural numbers $\aleph_{0}$ (see Asperó and Schindler [20]) - the maximum number with physical meaning in the universe is just SIDE.

We are, therefore, facing an ontological ${ }^{3}$ economic framework on a subplankian scale. It promises to be robust at high momentum transmitted to the package / particle as in nature. The huge number of bubbles forming the particles - indeed a mini universe each - gives material support to superpositions and qubits. These rules can be refined, given sufficient computational power and programming support ${ }^{4}$, and evaluated if they in fact allow building a predictive, bona fide, theory.

\section{References}

[1] Wheeler, J.A. Information, physics, quantum: the search for links, In Complexity, entropy and the physics of information (ed. W. Zurek). Reading, MA: Addison-Wesley (1990).

[2] Zuse, K. Rechnender raum, Elektronische Datenverarbeitung, 8, 336-344 (1967).

[3] Feynman, R. The character of the physical law, MIT, ISBN 0262560038 (1967).

[4] Gardner, M. The fantastic combinations of John Conway's new solitaire game "life", Sci. Am. 223, 120-123 (1970).

[5] Minsky M. Cellular vacuum, Int. J. Theor. Phys. 21: 537-551 (1982).

[6] Margolus N. Universal cellular automata based on the collisions of soft spheres, Adamatzky A. (eds) Collision-Based Computing. Springer, London (2002).

[7] Wolfram, S. A new kind of science, Wolfram Media, \{23-60\}, 112, and 865-866 (2002).

[8] Fredkin E. An introduction to digital philosophy. Int. J. Theor. Phys. 42 (2): 189-247 (2003).

[9] Elze, H.T. Action principle for cellular automata and the linearity of quantum mechanics, Phys. Rev. A 89012111 (2014).

[10] H.-T Elze Qubit exchange interactions from permutations of classical bits, Int. J. Quant. Info., 17 (08), 1941003 (2019).

[11] Sciarretta, A. A local-realistic model of quantum mechanics based on a discrete spacetime, Found. Phys. 48 (1), 60-91, (2018).

\footnotetext{
${ }^{3}$ Ontology is actually an always receding rule marking the frontier of the unfathomable.

${ }^{4} \mathrm{An}$ implementation under development is accessible in Reference [21]
} 
[12] 't Hooft, G. The cellular automaton interpretation of quantum mechanics, In: van Beijeren, H., et al. (eds.) Fundamental Theories of Physics. Springer, Berlin (2016).

[13] Smith, W.D. Charge quantization, the topology and 3-dimensionality of the universe, and abolishing monopoles, https://www.semanticscholar.org (2000).

[14] Lachieze-Rey M., and J.P. Luminet Cosmic topology, Phys. Rept. 254 (1995).

[15] Case, J., D. Rajan, and A. Shende Spherical wave front generation in lattice computers, Int. J. Comput. Inf. (1994).

[16] G. J. Chaitin, Algorithmic Information Theory, in IBM Journal of Research and Development, 21, no. 4, pp. 350-359 (1977).

[17] Itô D. Cohesive force of electron and Nambu's mass formula, Prog. Theor. Phys. 47, no. 3 (1972).

[18] Hofer, W.A. Elements of physics for the 21st century, J. Phys.: Conf. Ser. 504, 012014 (2014).

[19] Assis, A.K.T. Deriving gravitation from electromagnetism, Can. J. Phys. 70, 330-340 (1992).

[20] Asperó, D., R. Schindler Martin's Maximum implies Woodin's axiom (*). Annals of Mathematics, 193 (3), 793-835 (2021).

[21] Furtado Neto, A. It from bit - a concrete attempt, GitHub repository, https://github.com/automaton3d/automaton, (2021). 


\section{Appendix: source code}

Here are gathered all the rules of information exchange between cells of the cellular automaton. They are the essence of this work, intended to possess an axiomatic character.

I have used a C-like notation instead of traditional algorithm convention for clearness. The parameter ORDER comes from Section 2.

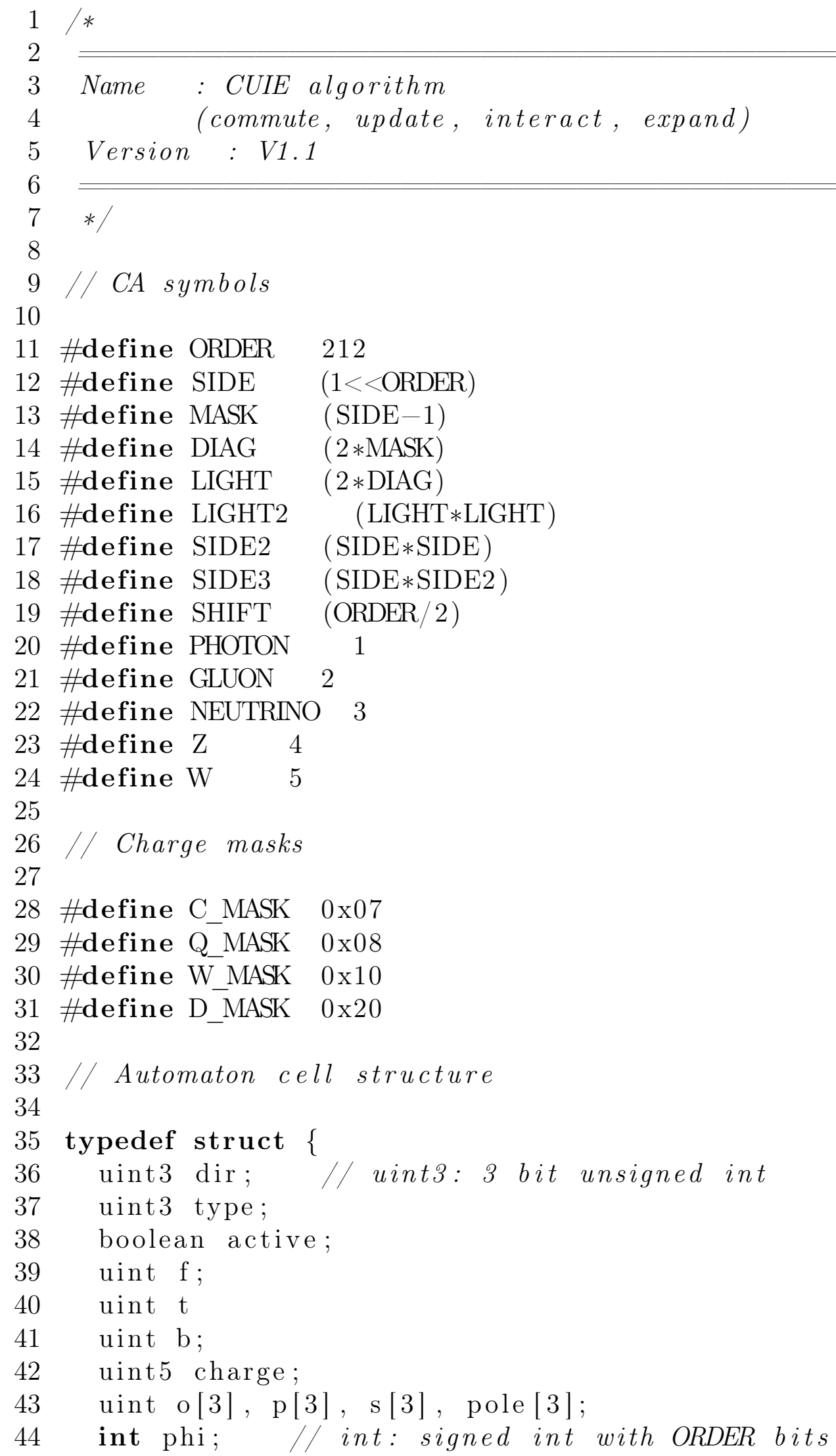


51 Cell;

52

53

54

55

56
57

57

58

59

60

61

62

63

64

65

66

67

68

69

70

71

72

73

74

75

76

77

78

79

80

81

82

83

84

85

86

87

88

89

90

91

92

93

94

\#define $\mathrm{S}$

*

*

//

//

$/ 1$

uint noise; // uint: unsigned int with ORDER bits

uint 4 code;

uint synch;

int sine, cosine;

uint ctrl;

\section{Macros}

\#define ISNULL $(\mathrm{v}) \quad(\mathrm{v}[0]==0 \quad \& \& \quad \mathrm{v}[1]==0 \quad \& \& \quad \mathrm{v}[2]==0)$

\#define $\operatorname{ISEQUAL}(\mathrm{v}, \mathrm{u}) \quad(\mathrm{v}[0]==\mathrm{u}[0] \quad \& \& \mathrm{v}[1]==\mathrm{u}[1] \quad \& \& \mathrm{v}[2]==\mathrm{u}[2])$

\#define RESET $(\mathrm{v}) \quad\{\mathrm{v}[0]=0 ; \mathrm{v}[1]=0 ; \mathrm{v}[2]=0 ;\}$

\#define $\operatorname{COPY}(\mathrm{u}, \mathrm{v}) \quad\{\mathrm{u}[0]=\mathrm{v}[0] ; \mathrm{u}[1]=\mathrm{v}[1] ; \mathrm{u}[2]=\mathrm{v}[2] ;\}$

\#define MOD2 $(\mathrm{v}) \quad(\mathrm{v}[0] * \mathrm{v}[0]+\mathrm{v}[1] * \mathrm{v}[1]+\mathrm{v}[2] * \mathrm{v}[2])$

\#define $\operatorname{nextV}(\mathrm{c}) \quad\{\mathrm{c} \rightarrow$ type\&0x02 $? \mathrm{c}-(\operatorname{SIDE} 3 *(\operatorname{SIDE} 2-1)): \mathrm{c}+\operatorname{SIDE} 3\}$

\#define CELL

$\operatorname{sizeof}(\mathrm{Cell})$

(SIDE/2)

Cell $*$ stable,$*$ draft

* Same code for all cells.

void main () \{

//////// STEP1: COMMUTE

// Copy all variables

stable $\rightarrow$ t $=$ draft $\rightarrow$ t;

stable $\rightarrow$ dir $=$ draft $\rightarrow$ dir ;

stable $\rightarrow$ charge $=$ draft $\rightarrow$ charge $;$

stable $\rightarrow$ code $=$ draft $\rightarrow$ code $;$

stable $\rightarrow$ noise $=$ draft $\rightarrow$ noise

stable $\rightarrow$ b $=$ draft $\rightarrow$ b

stable $\rightarrow$ synch $=$ draft $\rightarrow$ synch $;$

stable $\rightarrow \mathrm{f}=$ draft $\rightarrow \mathrm{f}$;

COPY (stable $\rightarrow$ p , draft $\rightarrow$ p);

COPY (stable $\rightarrow$ s, draft $\rightarrow$ s);

COPY (stable $\rightarrow$ o, draft $\rightarrow$ o);

COPY (stable $\rightarrow$ pole, draft $\rightarrow$ pole $)$;

// Commute roles

stable $\rightarrow$ active $=$ false

draft $\rightarrow$ active $=$ true;

Cell *temp $=$ draft;

draft $=$ stable;

stable = temp; 


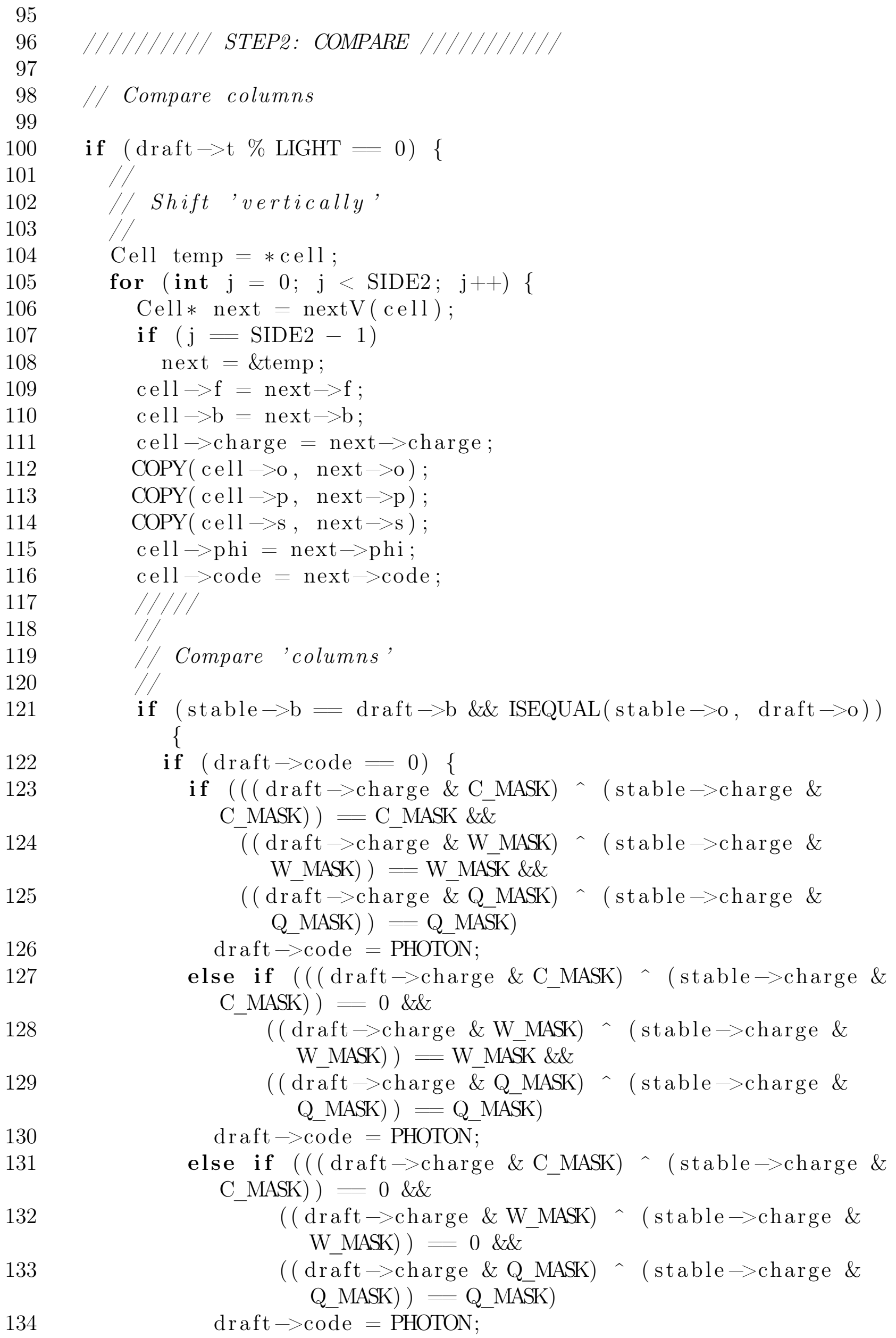


135

136

137

138

139

140

141

142

143

144

145

146

147

148

149

150

151

152

153

154

155

156

157

158

159

160

161

162

163

164

165

166

167

168

169

170

171

172

173

174

175

else if $((($ draft $\rightarrow$ charge \& C_MASK $) \wedge$ ( stable $\rightarrow$ charge \& C_MASK $))=0$ \&\&

$(($ draft $\rightarrow$ charge $\&$ W_MASK $) \wedge($ stable $\rightarrow$ charge $\&$ W_MASK) $)=0 \& \&$

$(($ draft $\rightarrow$ charge \& Q MASK $) \wedge($ stable $\rightarrow$ charge \& Q_MASK) $)=$ Q_MASK)

draft $\rightarrow$ code $=$ PHOTON;

else if $((($ draft $\rightarrow$ charge \& C_MASK $)$ ^ (stable $\rightarrow$ charge \& C_MASK $))=$ C_MASK \&\&

$(($ draft $\rightarrow$ charge $\&$ W_MASK $) \wedge($ stable $\rightarrow$ charge $\&$ W_MASK $))=0$ \&\&

$(($ draft $\rightarrow$ charge \& Q_MASK $) \wedge($ stable $\rightarrow$ charge \& Q_MASK) $)=$ Q_MASK)

$\mathrm{draft} \rightarrow$ code $=$ PHOTON;

else if $((($ draft $\rightarrow$ charge $\&$ C_MASK $) \wedge($ stable $\rightarrow$ charge \& C_MASK $))=$ C_MASK \&\&

$(($ draft $\rightarrow$ charge $\&$ W_MASK $) \wedge($ stable $\rightarrow$ charge $\&$ W_MASK $)=0 \& \&$

$(($ draft $\rightarrow$ charge \& Q MASK $)$ ( stable $\rightarrow$ charge \& Q_MASK) $)=0$ )

draft $\rightarrow$ code $=$ PHOTON;

/

if $($ draft $\rightarrow$ code $!=0)$

$\mathrm{draft} \rightarrow \mathrm{f}++$;

\}

else if $($ draft $\rightarrow$ code $=$ stable $\rightarrow$ code $)\{$

draft $\rightarrow \mathrm{f}++$;

\}

\}

cell $=\operatorname{nextV}($ cell $)$

\}

\}

/////// STEP3: REPLICATE /////////

// Once each light step

$/ /$

if $($ draft $\rightarrow$ t \% LIGHT $=0)\{$

$/ /$

// Copy only variables that changed in compare()

/

stable $\rightarrow \mathrm{f}=$ draft $\rightarrow \mathrm{f}$;

stable $\rightarrow$ code $=$ draft $\rightarrow$ code

\}

/////// STEP4: INTERACT /////////

// Once each light step

$/ /$

if $(\mathrm{draft} \rightarrow \mathrm{t} \%$ LIGHT $=0)\{$ 
176

177

178

179

180

181

182

183

184

185

186

187

188

189

190

191

192

193

194

195

196

197

198

199

200

201

202

203

204

205

206

207

208

209

210

211

212

213

214

215

216

217

218

219

int $\operatorname{sig} 1=(($ stable $\rightarrow$ charge $\wedge$ draft $\rightarrow$ charge $) \&$ C_MASK $)=0$ $\& \&$

$($ draft $\rightarrow$ charge \& C_MASK $)=0 \& \&$

$\left(\left(\right.\right.$ stable $\rightarrow$ charge ${ }^{-}$draft $\rightarrow$ charge $) \&$ Q_MASK $)=0$ \&\&

$($ draft $\rightarrow$ charge \& Q_MASK $)=$ Q_MASK \& \&

$(($ stable $\rightarrow$ charge $\wedge$ draft $\rightarrow$ charge $)$ \& D_MASK $)=0$ \&\&

$($ draft $\rightarrow$ charge \& D_MASK $)=0$;

int $\operatorname{sig} 2=(($ stable $\rightarrow$ charge $\wedge$ draft $\rightarrow$ charge $) \&$ C_MASK $)=0$

$\& \&$

$($ draft $\rightarrow$ charge \& C_MASK $)=$ C_MASK \&\&

$\left(\left(\right.\right.$ stable $\rightarrow$ charge ${ }^{\wedge}$ draft $\rightarrow$ charge $) \&$ Q_MASK $)=0$ \&\&

$($ draft $\rightarrow$ charge \& Q_MASK $)=0 \& \&$

$\left(\left(\right.\right.$ stable $\rightarrow$ charge ${ }^{\wedge} \mathrm{draft} \rightarrow$ charge $) \&$ D_MASK $)=0$ \&\&

$(\mathrm{draft} \rightarrow$ charge \& D_MASK $)=$ D_MASK;

int $\operatorname{sig} 3=(($ stable $\rightarrow$ charge $\wedge$ draft $\rightarrow$ charge $) \&$ C_MASK $)=0$ $\& \&$

$($ draft $\rightarrow$ charge \& C_MASK) $!=0$ \&\&

$($ draft $\rightarrow$ charge \& C_MASK) $!=$ C_MASK \&\&

$\left(\left(\right.\right.$ stable $\rightarrow$ charge ${ }^{\wedge}$ draft $\rightarrow$ charge $) \&$ Q_MASK $)=0$;

int $\mathrm{c} 1=(($ stable $\rightarrow$ charge $\wedge$ draft $\rightarrow$ charge $) \&$ Q_MASK $)=0$ \&\& $(($ stable $\rightarrow$ charge $\wedge \mathrm{draft} \rightarrow$ charge $) \&$ W_MASK $)=0$ \&\& sig 1

$$
=\operatorname{sig} 2 \text {; }
$$

int $\mathrm{c} 2=(($ stable $\rightarrow$ charge $\wedge$ draft $\rightarrow$ charge $) \&$ Q_MASK $) !=0 \& \&$ $(($ stable $\rightarrow$ charge $\wedge$ draft $\rightarrow$ charge $) \&$ W_MASK $) !=0$ \&\& $\operatorname{sig} 1 !=$ $\operatorname{sig} 2$

int $\mathrm{c} 3=($ stable $\rightarrow$ charge $\&$ D_MASK $)=0 \& \&($ stable $\rightarrow$ charge $\&$ W_MASK $)=0$ \&\&

$((\overline{\text { stable }} \rightarrow$ charge $\wedge$ draft $\rightarrow$ charge $) \&$ W_MASK $) \quad !=0$;

int $\mathrm{c} 4=($ stable $\rightarrow$ charge \& D_MASK $)=$ D_MASK \&\&

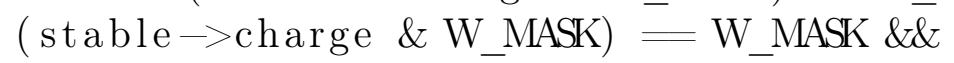

$(($ stable $\rightarrow$ charge $\wedge \overline{\mathrm{d}}$ raft $\rightarrow$ charge $) \&$ W_MASK $) !=0$;

$/ /$

if $(\operatorname{ISNULL}($ stable $\rightarrow$ pole $))$

COPY $($ draft $\rightarrow$ pole, stable $\rightarrow$ p) ;

$1 /$

// Play pseudo dices

if

if $($ stable $\rightarrow$ noise $>$ abs (stable $\rightarrow$ phi) \&\&

$\mathrm{draft} \rightarrow$ noise $>$ abs $(\mathrm{draft} \rightarrow$ phi $) \& \&$

$($ ! ISNULL $($ stable $\rightarrow$ p $) \quad||$ ! ISNULL $($ draft $\rightarrow$ p $))) \quad\{$

if $((($ stable $\rightarrow$ charge $\wedge \mathrm{draft} \rightarrow$ charge $) \&$ D_MASK $)=0)\{$

// Same sector?

$/ /$

if $($ stable $\rightarrow \mathrm{f}=1 \& \&$ draft $\rightarrow \mathrm{f}=1)\{$

/ $F x F$

if $((($ stable $\rightarrow$ charge $\wedge$ draft $\rightarrow$ charge $) \&$ Q_MASK $) !=0) \quad\{$ // Annihilation? 
stable $\rightarrow \mathrm{b}=($ stable $\rightarrow \mathrm{b} * \mathrm{draft} \rightarrow \mathrm{b}) \%$ SIDE2 ; // ??? erro ???

$$
\mathrm{draft} \rightarrow \mathrm{b}=\text { stable } \rightarrow \mathrm{b} \text {; }
$$

Reissue R1 and R2 from this

$$
\operatorname{RESET}(\text { stable } \rightarrow \text { pole }) ; / / \text { ??? }
$$

\}

$$
\text { COPY }(\text { draft } \rightarrow \text { pole, stable } \rightarrow \text { p) ; }
$$

else if $(\operatorname{sig} 1|| \operatorname{sig} 2|| \operatorname{sig} 3)\{$<smiles>C1=C2CC=C2CC1</smiles>

// Similar?

$\gamma /$

\section{//Cohesion}

if $($ stable $\rightarrow$ b $!=$ draft $\rightarrow$ b) \{ stable $\rightarrow b=($ stable $\rightarrow b *$ draft $\rightarrow b) \%$ SIDE2 ;

\} $\mathrm{draft} \rightarrow \mathrm{b}=$ stable $\rightarrow \mathrm{b}$;

$$
s 1<->s^{2}
$$

int temp;

temp $=$ stable $\rightarrow$ s $[0] ;$

$\mathrm{draft} \rightarrow \mathrm{s}[0]=$ stable $\rightarrow$ s $[0] ;$

stable $\rightarrow$ s $[0]=$ temp ;

temp $=$ stable $\rightarrow$ s $[1]$;

draft $\rightarrow$ s [1] $=$ stable $\rightarrow$ s [1];

stable $\rightarrow$ s $[1]=$ temp;

temp $=$ stable $\rightarrow$ s $[2]$;

$\mathrm{draft} \rightarrow \mathrm{s}[2]=$ stable $\rightarrow$ s $[2] ;$

stable $\rightarrow$ s $[2]=$ temp ;

\section{$/ /$ \\ // Reissue R1 from pole(R1) and R2 from pole(R2)}

$\operatorname{RESET}($ stable $\rightarrow$ o) ;

$\operatorname{RESET}($ draft $\rightarrow$ o $) ; / /$ ? ? ?

COPY (draft $\rightarrow$ pole, stable $\rightarrow$ p) ; //???

\}

else if $($ stable $\rightarrow \mathrm{f}>1 \& \& \mathrm{draft} \rightarrow \mathrm{f}>1)\{$

$$
/ / / B \times B
$$

if $((($ stable $\rightarrow$ charge $\wedge \sim$ draft $\rightarrow$ charge $) \&$ C_MASK $)=0$ $\& \&$ stable $\rightarrow$ code $=$ draft $\rightarrow$ code $\& \&$ draft $\rightarrow$ code $=$ GLUON $)$ \{

gluon-gluon? 
267

268

269

270

271

272

273

274

275

276

277

278

279

280

281

282

283

284

285

286

287

288

289

290

291

292

293

294

295

296

297

298

299

300

301

302

303

304

305

306

307

308

309

310

311

312

313

314

315

\section{Swap colors}

int temp $=$ stable $\rightarrow$ charge \& C_MASK;

stable $\rightarrow$ charge $\&=\sim$ C_MASK;

stable $\rightarrow$ charge $\mid=($ draft $\rightarrow$ charge \& C_MASK $)$;

draft $\rightarrow$ charge $\&=\sim$ C_MASK;

draft $\rightarrow$ charge $\mid=$ temp

$/ /$

Reissue R1 from pole(R1)

$\operatorname{RESET}($ stable $\rightarrow$ o ) ;

$\mathrm{draft} \rightarrow \mathrm{draft} \rightarrow \mathrm{dir}=0 ; / /$ replicar !!!

$\mathrm{draft} \rightarrow \mathrm{t}=0 ; / /$ replicar !!!

\}

else if (!ISNULL $($ stable $\rightarrow$ p) \&\& !ISNULL $($ draft $\rightarrow$ p $))\{$

if $\left(\begin{array}{llll|l|ll}\mathrm{c} 1 & || & \mathrm{c} 2 & \| & \mathrm{c} 3 & || & \mathrm{c} 4\end{array}\right)$

\{

// chiral?

$/ /$ Reissue $R 1$ and R2 from cp1

$/ /$

$\mathrm{draft} \rightarrow \mathrm{b}=$ stable $\rightarrow$ b

$\operatorname{RESET}($ stable $\rightarrow$ o) ; 
316

317

318

319

320

321

322

323

324

325

326

327

328

329

330

331

332

333

334

335

336

337

338

339

340

341

342

343

344

345

346

347

348

349

350

351

352

353

354

355

356

357

358

359

360

361

362

363

364

365

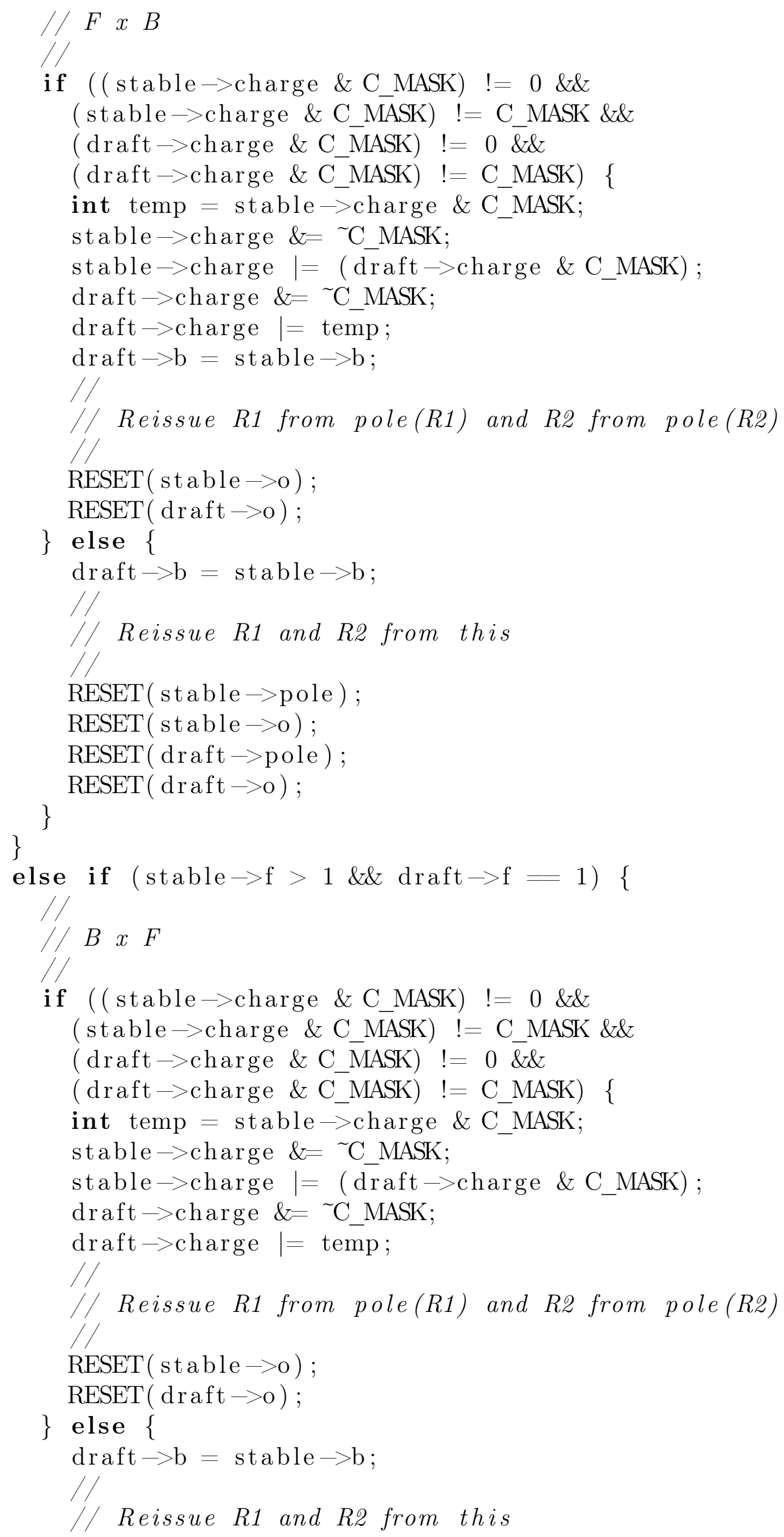


366

367

368

369

370

371

372

373

374

375

376

377

378

379

380

381

382

383

384

385

386

387

388

389

390

391

392

393

394

395

396

397

398

399

400

401

402

403

404

405

406

407

408

409

410

411

412

413

414

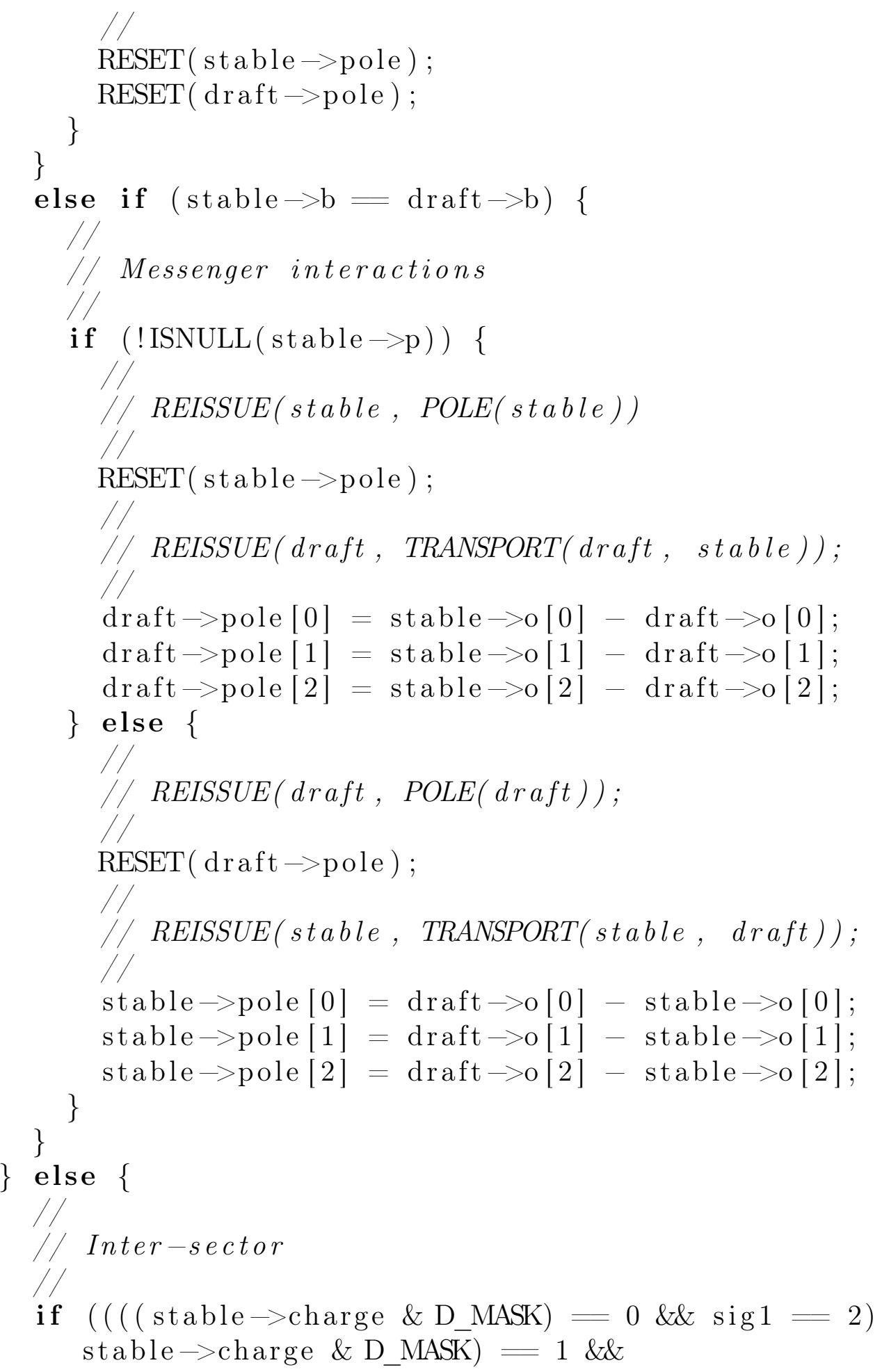


415

416

417

418

419

420

421

422

423

424

425

426

427

428

429

430

431

432

433

434

435

436

437

438

439

440

441

442

443

444

445

446

447

448

449

450

451

452

453

454

455

456

457

458

459

460

461

462

463

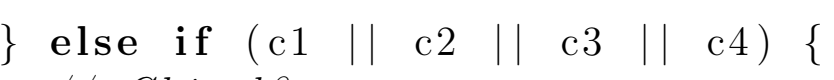

// Chiral?

int temp $=$ stable $\rightarrow$ charge \& W_MASK;

stable $\rightarrow$ charge $\&=$ W_MASK;

stable $\rightarrow$ charge $\mid=($ d $\bar{r}$ aft $\rightarrow$ charge \& W_MASK $)$;

draft $\rightarrow$ charge $\&=$ W_MASK;

draft $\rightarrow$ charge $\mid=$ temp

$/ /$

// Reissue R1 and R2 from this

$\operatorname{RESET}($ stable $\rightarrow$ pole $)$;

$\operatorname{RESET}($ draft $\rightarrow$ pole $)$;

\}$^{\}^{3}}$

$/ / / / / / /$ STEP5: EXPAND

// Update tracking info

$/ /$

if $(\mathrm{draft} \rightarrow \mathrm{ctrl}>0)\{$

// Track decay

$/ /$

draft $\rightarrow$ phi $*=(1-1 /(2 *$ draft $\rightarrow$ t $))$;

//

// Minsky circle algorithm

\section{$/ 1$}

int $\mathrm{xNew}=\mathrm{draft} \rightarrow \operatorname{cosine}-(\mathrm{draft} \rightarrow$ sine $>$ SHIFT $)$;

int $\mathrm{yNew}=\mathrm{draft} \rightarrow$ sine $+(\mathrm{draft} \rightarrow \operatorname{cosine} \gg$ SHIFT $)$;

draft $\rightarrow$ cosine $=$ xNew;

draft $\rightarrow$ sine $=$ yNew;

$/ /$

$\mathrm{draft} \rightarrow \mathrm{ctrl}-\ldots$

\}

Spread cell contents if not empty

$/ /$

if $(\mathrm{draft} \rightarrow \mathrm{f}>0)\{$

$\mathrm{draft} \rightarrow \mathrm{t}++$;

Cell* neighbor ;

$/ / /$ Momentum evolution

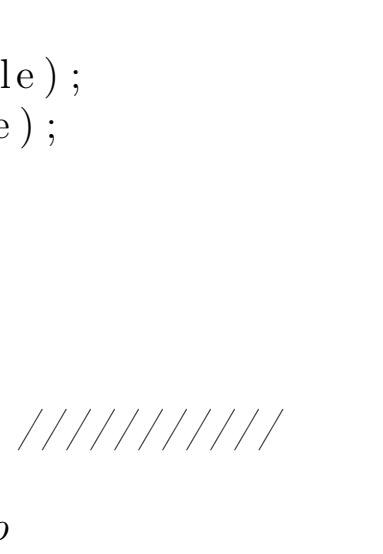

if $($ ! ISNULL $(\mathrm{draft} \rightarrow \mathrm{p}) \& \&$ draft $\rightarrow$ t $*$ draft $\rightarrow$ t $>$ draft $\rightarrow$ synch $)\{$

$$
\text { // Select the only path for momentum }
$$


464

465

466

467

468

469

470

471

472

473

474

475

476

477

478

479

480

481

482

483

484

485

486

487

488

489

490

491

492

493

494

495

496

497

498

499

500

501

502

503

504

505

506

507

508

509

510

511

512 for (int $\operatorname{dir}=0 ; \operatorname{dir}<6 ; \operatorname{dir}++)\{$ char $\operatorname{vdir}[3]=\{0,0,0\}$;

neighbor $=$ getPointer $($ dir, draft , $($ char $*) \operatorname{vdir})$; $/ /$

// Predict next pole value

char pole [3];

COPY (pole, draft $\rightarrow$ pole $)$;

pole $[0]-=\operatorname{vdir}[0]$;

pole $[1]-=\operatorname{vdir}[1]$;

pole $[2]-=\operatorname{vdir}[2] ;$

$/ /$

Test if pole shrunk

if $(\mathrm{MOD} 2($ pole $)<\operatorname{MOD} 2($ draft $\rightarrow$ pole $))\{$

neighbor $\rightarrow$ pole $[0]=$ pole $[0]$;

neighbor $\rightarrow$ pole $[1]=$ pole $[1]$;

neighbor $\rightarrow$ pole $[2]=$ pole $[2]$;

neighbor $\rightarrow \mathrm{t}=\mathrm{draft} \rightarrow \mathrm{t}$;

neighbor $\rightarrow \mathrm{f}=\mathrm{draft} \rightarrow \mathrm{f}$;

neighbor $\rightarrow$ b $=$ draft $\rightarrow$;

neighbor $\rightarrow$ charge $=$ draft $\rightarrow$ charge

neighbor $\rightarrow$ o $[0]=\operatorname{draft} \rightarrow$ o[0] $+\operatorname{vdir}[0] ;$

neighbor $\rightarrow$ o $[1]=\operatorname{draft} \rightarrow$ o[1] $+\operatorname{vdir}[1]$;

neighbor $\rightarrow$ o[2] $=\operatorname{draft} \rightarrow \mathrm{O}[2]+\operatorname{vdir}[2]$;

//

COPY (neighbor $\rightarrow$ s, draft $\rightarrow$ s $)$;

COPY (neighbor $\rightarrow$ p, draft $\rightarrow$ p);

$1 /$

Synchronize to keep inside spherical wavefront

//

neighbor $\rightarrow$ synch $=$ LIGHT2 $*$ MOD2(neighbor $\rightarrow$ o) ;

int $\mathrm{t}=$ neighbor $\rightarrow \mathrm{t}$;

if (ISNULL (neighbor $\rightarrow$ pole $))\{$

neighbor $\rightarrow \mathrm{t}=0$;

neighbor $\rightarrow$ synch $=$ LIGHT;

neighbor $\rightarrow \mathrm{f}=1$;

neighbor $\rightarrow \mathrm{b}=0$;

neighbor $\rightarrow$ code $=0$;

$\operatorname{RESET}($ neighbor $\rightarrow$ o) ;

This characterizes free propagation

COPY (neighbor $\rightarrow$ pole, neighbor $\rightarrow$ p) ;

\}

Keep only a single copy of momentum 
513

514

515

516

517

518

519

520

521

522

523

524

525

526

527

528

529

530

531

532

533

534

535

536

537

538

539

540

541

542

543

544

545

546

547

548

549

550

551

552

553

554

555

556

557

558

559

560

561

\section{$\operatorname{RESET}($ draft $\rightarrow$ p) ;}

When momentum moves, it casts a spherical perturbation

draft $\rightarrow$ t $=0$;

$\mathrm{draft} \rightarrow$ synch $=$ LIGHT;

draft $\rightarrow$ f $=1$;

$\mathrm{draft} \rightarrow \mathrm{b}=0$;

draft $\rightarrow$ code $=0$;

$\operatorname{RESET}($ draft $\rightarrow$ o ) ;

break ;

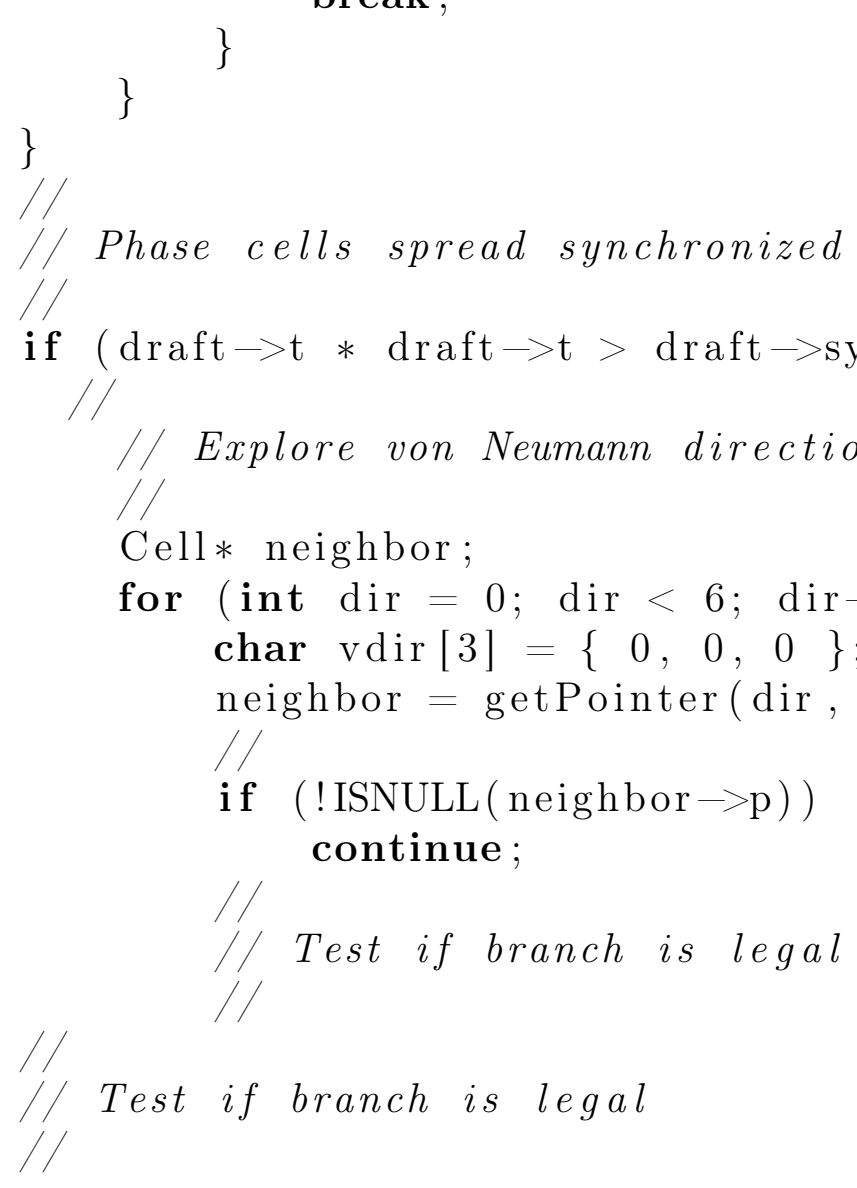

boolean allowed $=$ false;

Calculate new origin vector

int $\mathrm{x}=\mathrm{o}[0]+\mathrm{vdir}[0]$;

int $\mathrm{y}=\mathrm{o}[1]+\mathrm{vdir}[1]$;

int $\mathrm{z}=\mathrm{o}[2]+\mathrm{vdir}[2]$;

// Test for expansion

int $\mathrm{d} 1=\operatorname{MOD} 2(\mathrm{o})$;

int $\mathrm{d} 2=\mathrm{x} * \mathrm{x}+\mathrm{y} * \mathrm{y}+\mathrm{z} * \mathrm{z}$;

if $(\mathrm{d} 2<=\mathrm{d} 1)$ 


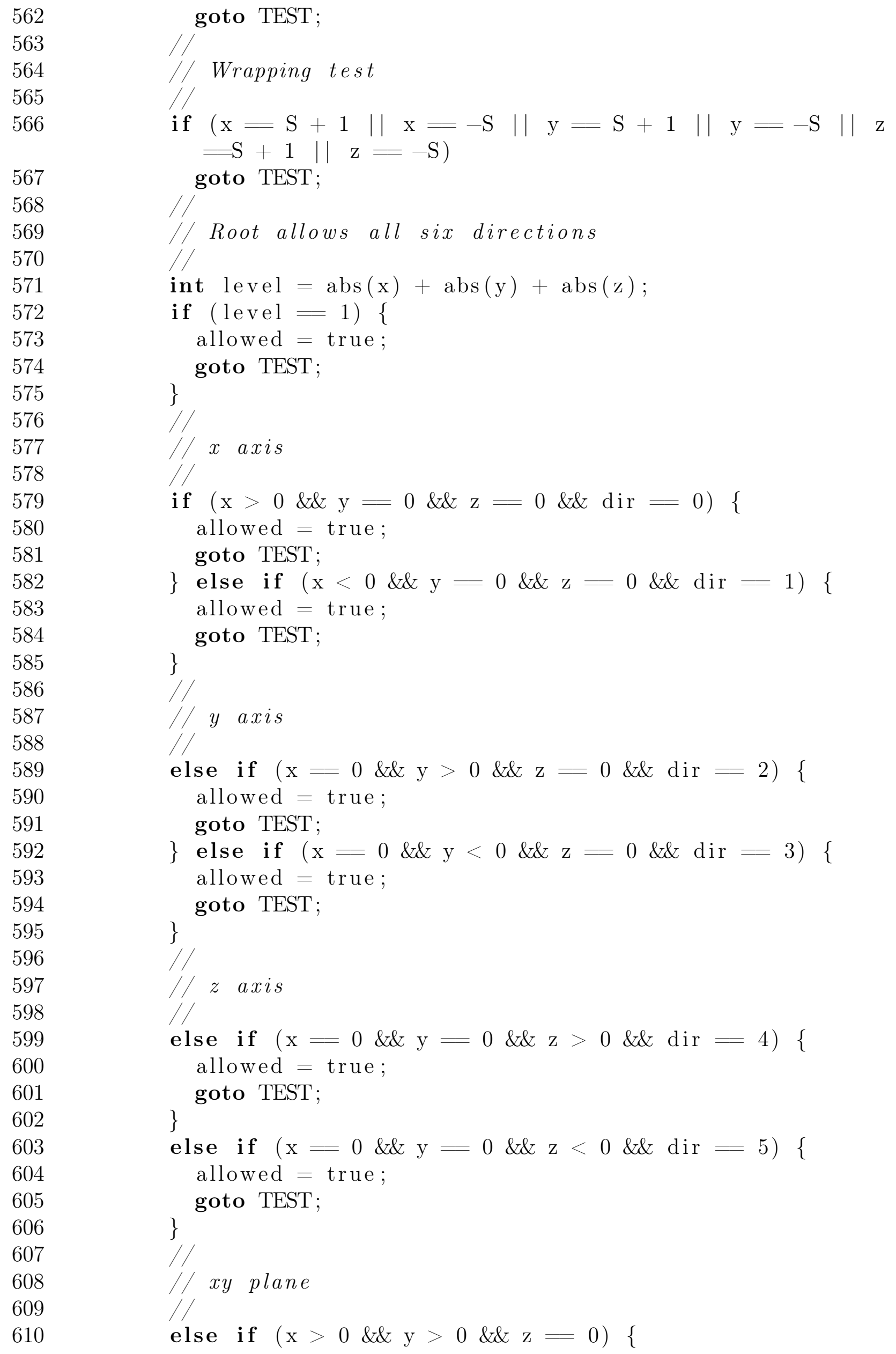


611

612

613

614

615

616

617

618

619

620

621

622

623

624

625

626

627

628

629

630

631

632

633

634

635

636

637

638

639

640

641

642

643

644

645

646

647

648

649

650

651

652

653

654

655

656

657

658

659

660

if (level \%2=1)

allowed $=(\operatorname{dir}=0 \& \&$ draft $\rightarrow$ dir $=2)$;

else

allowed $=($ dir $=2 \& \&$ draft $\rightarrow$ dir $=0)$;

else if $(\mathrm{x}<0$ \&\& $\mathrm{y}>0$ \&\& $\mathrm{z}=0)\{$

if (level \% $2=1$ )

allowed $=(\operatorname{dir}=1 \& \&$ draft $\rightarrow$ dir $=2)$;

else

allowed $=(\operatorname{dir}=2 \& \&$ draft $\rightarrow$ dir $=1)$;

\}

else if $(\mathrm{x}>0 \& \& \mathrm{y}<0 \& \& \mathrm{z}=0)\{$

if (level \% $2=1$ )

allowed $=(\operatorname{dir}=0$ \&\& draft $\rightarrow$ dir $=3)$;

else

allowed $=(\operatorname{dir}=3 \& \&$ draft $\rightarrow$ dir $=0)$;

\}

else if $(\mathrm{x}<0 \& \& \mathrm{y}<0 \& \& \mathrm{z}=0)\{$

if (level \% $2=1$ )

allowed $=(\operatorname{dir}=1$ \&\& draft $\rightarrow$ dir $=3)$;

else

allowed $=($ dir $=3 \& \&$ draft $\rightarrow$ dir $=1)$;

\}

//yz plane

else if $(\mathrm{x}=0 \& \& \mathrm{y}>0 \& \& \mathrm{z}>0)\{$

if (level \% $2=0)$

allowed $=(\operatorname{dir}=4 \& \&$ draft $\rightarrow$ dir $=2)$; else

allowed $=($ dir $=2 \& \&$ draft $\rightarrow$ dir $=4)$;

\}

else if $(\mathrm{x}=0$ \&\& $\mathrm{y}<0 \& \& \mathrm{z}>0)\{$

if (level \% $2=0)$

allowed $=(\operatorname{dir}=4 \& \&$ draft $\rightarrow$ dir $=3)$; else

allowed $=(\operatorname{dir}=3 \& \&$ draft $\rightarrow$ dir $=4)$;

\}

else if $(\mathrm{x}=0$ \&\& $\mathrm{y}>0$ \&\& $\mathrm{z}<0)\{$

if (level \% $2=0$ )

allowed $=(\operatorname{dir}=5 \& \&$ draft $\rightarrow$ dir $=2)$;

else

allowed $=($ dir $=2 \& \&$ draft $\rightarrow$ dir $=5)$;

\}

else if $(\mathrm{x}=0$ \&\& $\mathrm{y}<0 \& \& \mathrm{z}<0)\{$

if (level \% $2=0$ )

allowed $=(\operatorname{dir}=5 \& \&$ draft $\rightarrow$ dir $=3)$;

else

allowed $=(\operatorname{dir}=3 \& \&$ draft $\rightarrow$ dir $=5)$; 


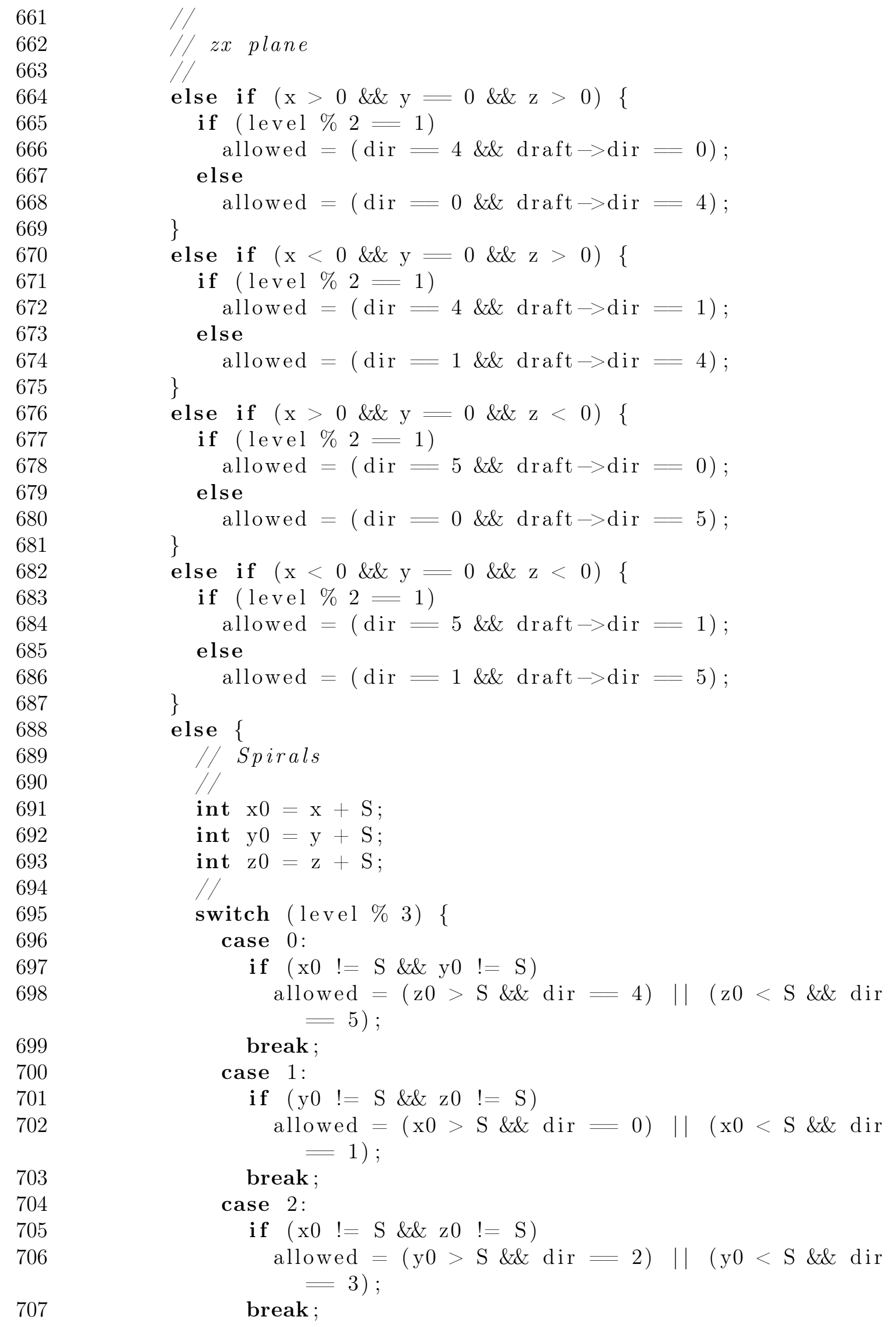




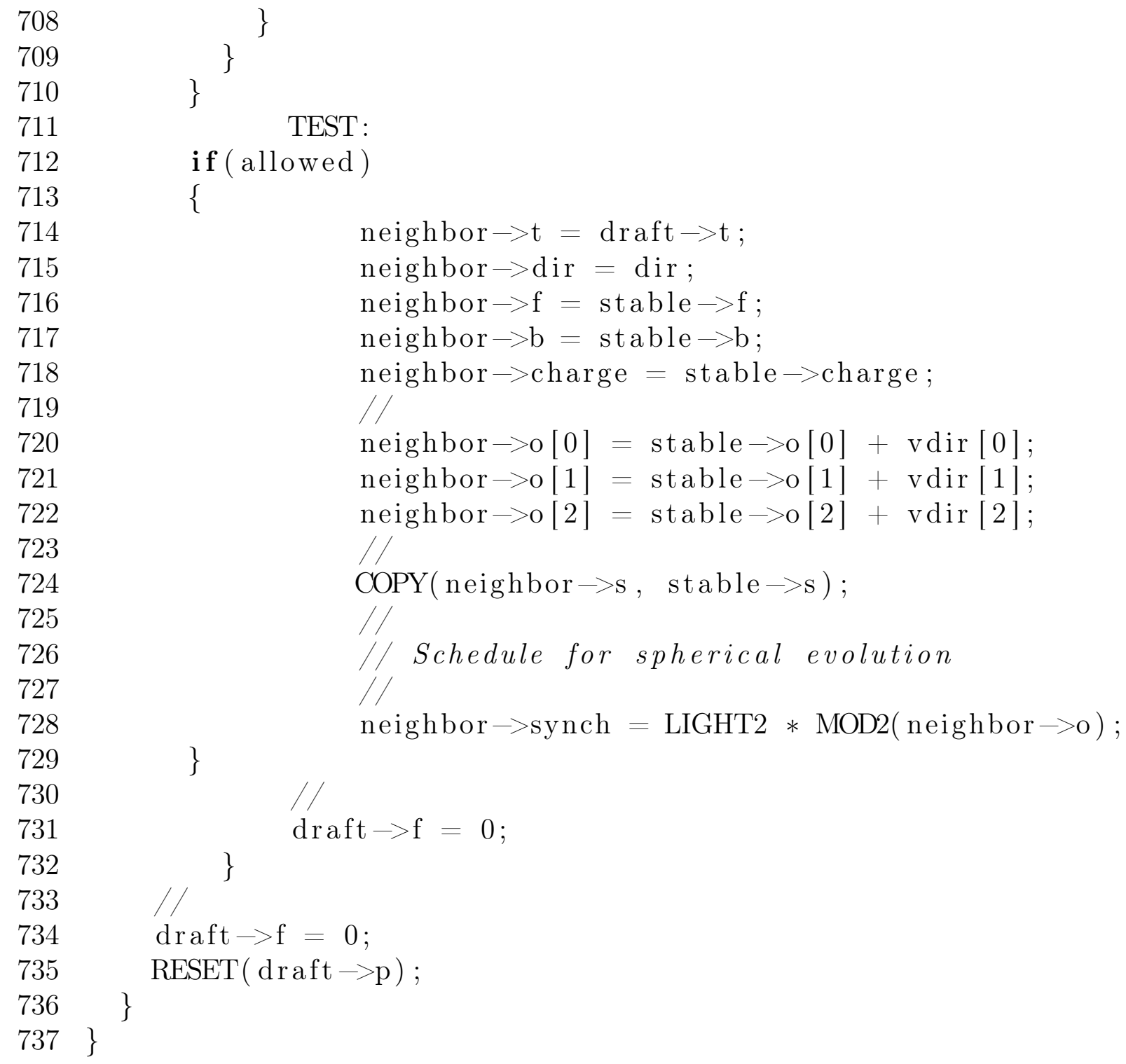

